\title{
One-step puncture and dilation with fine-gauge electrocautery dilator for endoscopic ultrasound-guided gallbladder drainage
}

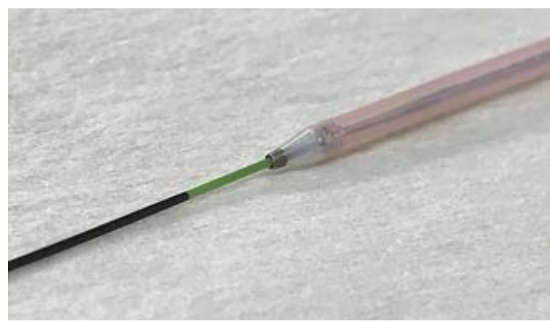

- Fig. 1 The novel fine-gauge electrocautery dilator (Fine 025, Medico's Hirata Inc., Osaka, Japan). The distal end of the outer dilator has a 3-Fr metal tip. This electrocautery dilator is wire-guided and coaxial with a 0.025 -inch guidewire. In addition, contrast medium injection is possible under guidewire loading.

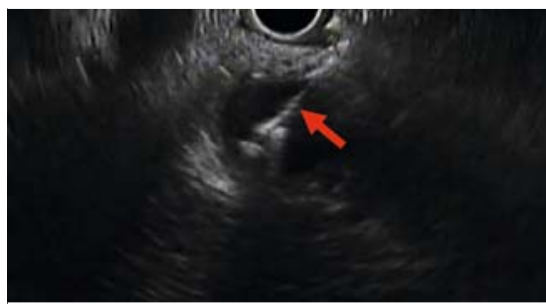

- Fig. 2 Endoscopic ultrasound image shows that the gallbladder was successfully punctured by the fine-gauge electrocautery dilator (red arrow).

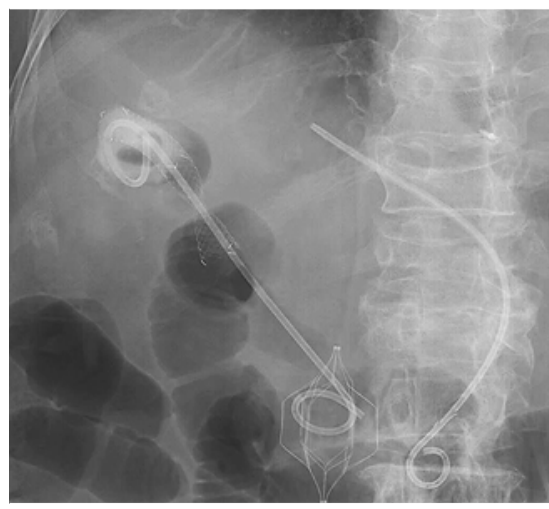

- Fig. 4 A 7-Fr double-pigtail plastic stent was placed within a fully covered metal stent.

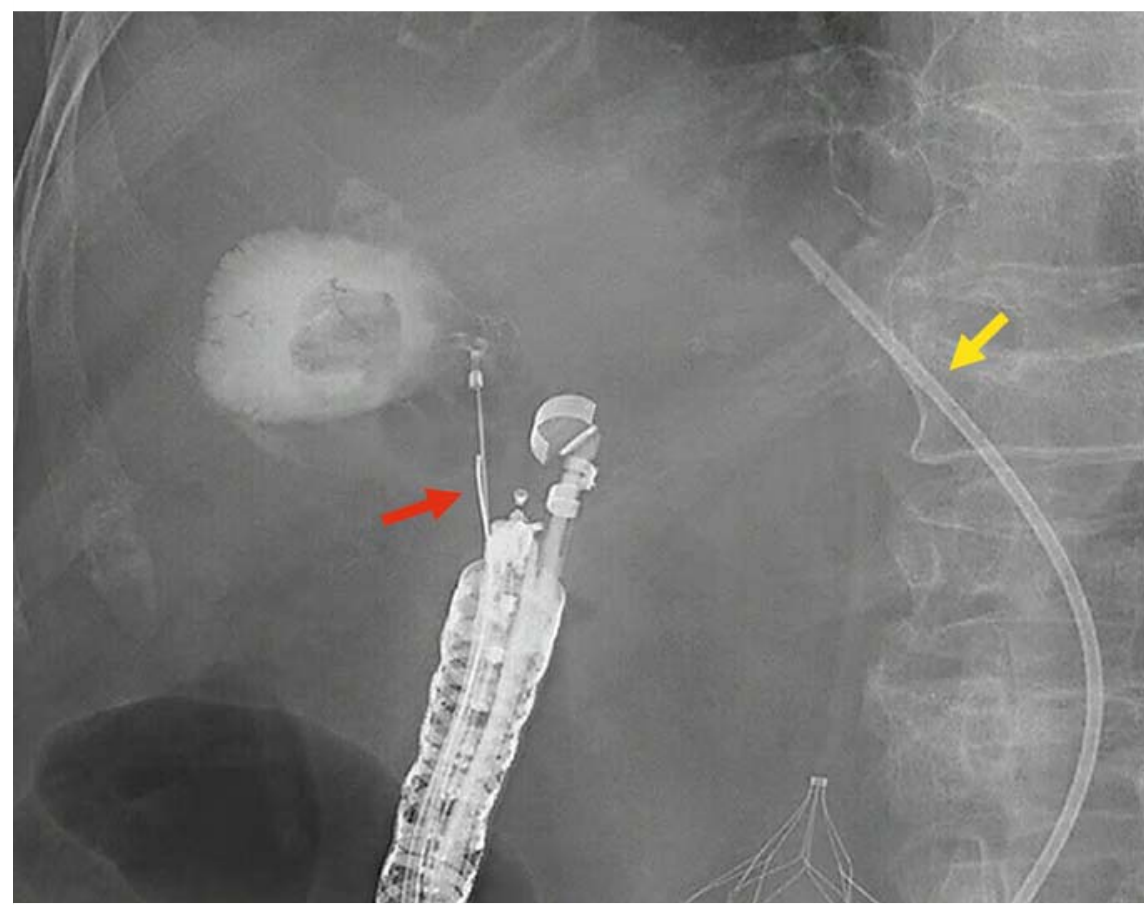

- Fig. 3 Contrast medium injection was possible under guidewire loading (red arrow). A plastic stent placed one and a half years earlier during endoscopic ultrasound-guided hepaticogastrostomy for the management of cholangitis was recognized (yellow arrow).

Recently the usefulness of a novel finegauge electrocautery dilator (Fine 025; Medico's Hirata Inc., Osaka, Japan) ( $\triangleright$ Fig. 1) has been reported for endoscopic ultrasound (EUS)-guided therapy [1-3]. Herein we describe a successful one-step puncture and tract dilation using this dilator during EUS-guided gallbladder drainage (EUS-GBD).

An 83-year-old woman was admitted to our hospital with recurrent gallstone cholecystitis. Considering her performance status, we decided to perform EUSGBD.

The gallbladder was visualized using an echoendoscope from the duodenum. A shorter procedure time and fewer device exchanges may be required to reduce bile leakage; however, one-step devices, such as the Hot AXIOS (Boston Scientific, Marlborough, MA), are not yet available for EUS-GBD in Japan. Therefore, we at- tempted to puncture the gallbladder and dilate the tract in one step using a fine-gauge electrocautery dilator (Fine 025) with a preloaded 0.025 -inch guidewire (VisiGlide 2; Olympus Medical Systems, Tokyo, Japan).

The gallbladder was successfully punctured with an electrocautery dilator ( $\triangleright$ Fig.2), and subsequent injection of contrast medium was possible under guidewire loading ( $\triangleright$ Fig.3). After the guidewire was inserted and coiled into the gallbladder under fluoroscopic guidance, the dilator was removed. A fully covered metal stent (diameter, $10 \mathrm{~mm}$; length, $6 \mathrm{~cm}$ ) (BONA stent; Standard SciTech Inc., Seoul, Korea) was successfully placed without additional tract dilation from the gallbladder into the duodenum. Finally, a 7-Fr double-pigtail plastic stent (length, $10 \mathrm{~cm}$ ) (Mediglobe $\mathrm{GmbH}$, Rosenheim, Germany) was passed from 


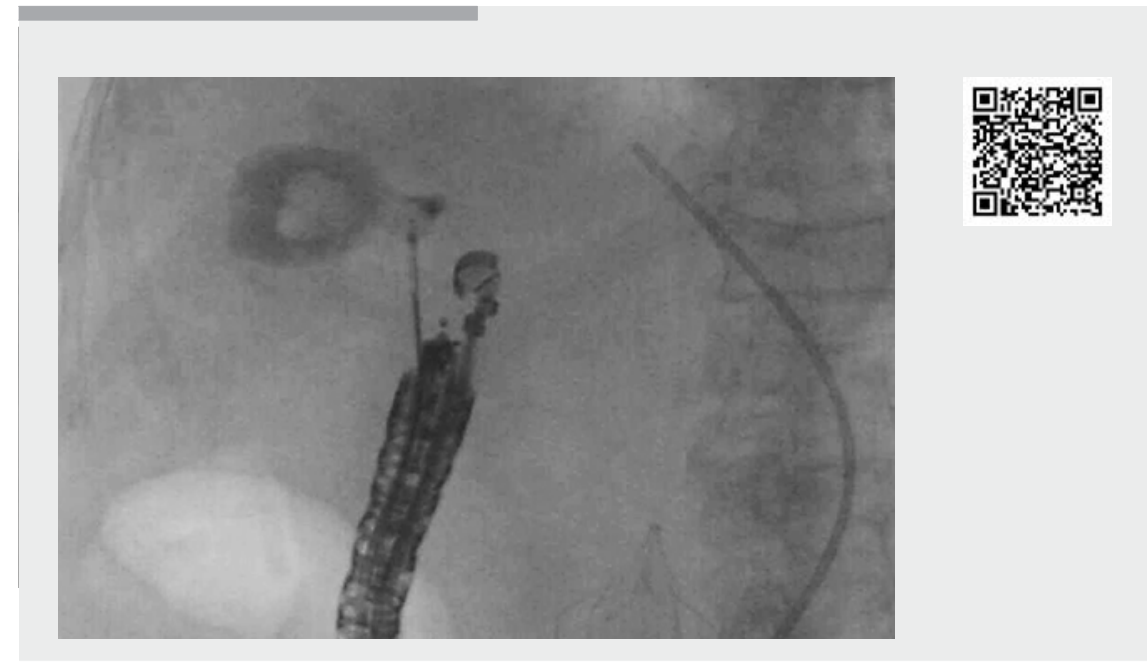

$\checkmark$ Video 1 Puncture and tract dilation in one step using a fine-gauge electrocautery dilator during endoscopic ultrasound-guided gallbladder drainage.

the gallbladder to the duodenum through the metal stent to prevent stent migration and food impaction (> Fig.4, - Video 1).

Our experience with this case suggests that puncture and tract dilation in one step using a fine-gauge electrocautery dilator is an option for EUS-GBD. Further studies involving many cases are needed to validate the safety and efficacy of this one-step procedure.

Endoscopy_UCTN_Code_TTT_1AS_2AD

\section{Competing interests}

The authors declare that they have no conflict of interest.

\section{References}

[1] Ogura T, Nakai Y, Iwashita T et al. Novel fine gauge electrocautery dilator for endoscopic ultrasound-guided biliary drainage: experimental and clinical evaluation study (with video). Endosc Int Open 2019; 7: E1652E1657

[2] Mandai K, Uno K, Yasuda K. Endoscopic ultrasound-guided pancreatic duct drainage using a novel fine-gauge electrocautery dilator. Endoscopy 2019; 51: E388-E389

[3] Ogura T, Nishioka N, Yamada M et al. Technical feasibility and safety of transluminal antegrade dilation for hepaticojejunostomy stricture using a novel fine-gauge electrocautery dilator (with video). Endosc Int Open 2020; 8: E733-E737

\section{Bibliography}

Endoscopy 2021; 53: E217-E218

DOI 10.1055/a-1244-9842

ISSN 0013-726X

published online 11.9.2020

(c) 2020. Thieme. All rights reserved.

Georg Thieme Verlag KG, Rüdigerstraße 14,

70469 Stuttgart, Germany Kenjiro Yasuda

Department of Gastroenterology, Kyoto Second Red Cross Hospital, Kyoto, Japan

\section{Corresponding author}

\section{Koichiro Mandai, MD}

Department of Gastroenterology, Kyoto Second Red Cross Hospital, 355-5 Haruobicho, Kamigyo-ku, Kyoto 602-8026, Japan Fax: +81-75-256-3451

mndkchr@gmail.com 